\title{
Non-life Insurance Mathematics
}

\author{
Makoto Yamazato
}

November, 2014

\begin{abstract}
In this work we describe the basic facts of non-life insurance and then explain risk processes. In particular, we will explain in detail the asymptotic behavior of the probability that an insurance product may end up in ruin during its lifetime. As expected, the behavior of such asymptotic probability will be highly dependent on the tail distribution of each claim.
\end{abstract}

MSC(2010): 60G07, 62P05; 97M30.

Keywords: Stochastic processes, actuarial mathematics, non-live insurance, collectice risk models, ruin probability, Cramér-Lundberg approximation.

1. University of the Ryukyus, Japan. 


\section{Introduction}

Insurance mathematics, which is also called actuarial mathematics, is studied with a mathematical viewpoint in Europe and the United States. There are scientific journals which mainly deal with actuarial mathematics (e.g. Scandinavian Actuarial Journal). However, it is not a very popular topic in the Japanese mathematical community. This may also be true for Peru. There is a professional qualification for this activity called actuary. An actuary has to design, price, and control the so called insurance commodities. They play a significant role in insurance companies as they make a final decision for the design of insurance commodities. Many mathematical tools are needed in order to perform this job. The main mathematical techniques required are related to probability theory and mathematical statistics.

In order to obtain this professional qualification, one must pass an examination. The examination is divided into three parts: life insurance, non-life insurance, and annuity. Life insurance mathematics is one of the basic exams and perhaps the most important one. A life insurance contract between the insurance company and a customer is a long time bound. Hence, the interest rate for bank deposits during a long time span and the life of a person may be considered as random phenomena. Therefore, we need to know their distribution. Regarding the life of a person this is the so called life expectancy (or death probability). Some special unique symbols are used in life insurance mathematics. These symbols are unique in this world and are not commonly seen in the mathematical world. But they have been standardized worldwide, and they are also used in non-life insurance and annuity. Therefore, in order to study insurance mathematics, one should be accustomed with the use of these symbols.

On the other hand, for non-life insurance, the period of contracts are relatively short. So, the influence of interest rates is neglected. Instead, two other factors are considered: the frequency of occurrence of accidents and their size. These can be regarded, respectively, as a probability law 
and a random variable with certain distribution function.

In this work, we describe the basic facts of non-life insurance and then explain risk processes, which are still an important research object in non-life insurance and applied probability. This is an application of the theory of stochastic processes. In particular, we will explain in detail the asymptotic behavior of the probability that an insurance product may end up in ruin during its lifetime. That is, given an initial investment amount set aside for payments and a premium rate per unit of time, we will discuss how to compute the probability that at some point in time the money amount of the total claims will be higher than the initial investment plus all the premium collected up to that point in time. As expected, the behavior of such asymptotic probability will be highly dependent on the tail distribution of each claim. We will call these classes light tail or heavy tailed depending on the probability of creating a large claim.

\section{Non-life (damage) Insurance}

There are many examples of non-life insurance: car insurance, weather related insurance, fire and earthquake insurance, aviation insurance, travel insurance, marine insurance, and so on. Let us consider for instance car insurance. Imagine a car owner. He may suffer a car accident. This accident is unpredictable, so it is a random phenomenon and, even, a rare event. Also the amount of money required for the repair of the car depends of the size of the accident and it is also unpredictable, another random phenomenon.

In this chapter, we summarize fundamental facts in non-life insurance necessary for the understanding of risk processes explained later on.

The request of insurance payment from a customer of a non-life insurance is called a claim. The total amount of claims in a prefixed unit time interval is the sum of the amounts of claims occurred in the time interval from all the customers. The number of claims and their 
amount in each term (or fixed time interval) are not constant. So, we consider each of these quantities as random variables.

\section{$2.1 \quad$ Number of claims}

First, we assume the homogeneity of an objective population (group) in the sense that all contracts are equal within a population for which each individual has the same risk. Assume that each one of $n$ contracts in a unit time interval may be the source of a claim independent of each other with given probability $p$. Then the probability that $k$ claims occur in one unit of time is given by the binomial distribution ${ }_{n} C_{k} p^{k}(1-p)^{n-k}$.

Let $N$ be a random variable which represents the number of claims in the unit time interval. The distribution of $N$ is the binomial distribution $B(n, p)$. If the number of contracts $n$ is very large and the probability $p$ of occurrence of the claim is very small, then the distribution of $N$ can be regarded via an approximation argument as the Poisson distribution. This is explained mathematically by the following argument. Assume $\lambda=n p$ and let $n \rightarrow \infty$. Then the distribution of $N$ converges to the Poisson distribution with parameter $\lambda$ since using the moment generating function formula for a binomial distribution we have

$$
M_{N}(\theta)=E\left(e^{\theta N}\right)=\left(1+\left(e^{\theta}-1\right) \lambda / n\right)^{n} \rightarrow \exp \left(\lambda\left(e^{\theta}-1\right)\right) .
$$

The right hand side is the moment generating function of the Poisson distribution with parameter $\lambda$ (denoted as $\operatorname{Po}(\lambda)$ ). For $N \sim \operatorname{Po}(\lambda)$ recall that we have $E(N)=V(N)=\lambda$.

If the objective population is not homogeneous, i.e., if contracts with various risks are mixed in the population, then the variance of $N$ is greater than its expectation $(V(N)>E(N))$. In such a case, the negative binomial distribution may fit as the distribution of $N$. The following is a mathematical explanation for this assertion. Let $Y_{\lambda} \sim \operatorname{Po}(\lambda)$, $(\lambda>0), W \sim \Gamma(\alpha, \beta)$ (i.e., the gamma distribution with parameters $\alpha$ and $\beta$ ) and assume $\left\{Y_{\lambda}\right\}$ and $W$ are independent. Let $N=Y_{W}$. This means the parameter of the Poisson random variable may vary according 
to the value of $W(\omega)$. In this context we have

$$
\begin{aligned}
P(N=k) & =\int_{0}^{\infty} P\left(Y_{\lambda}=k \mid W=\lambda\right) P(W \in d \lambda) \\
& =\int_{0}^{\infty} \frac{\lambda^{k}}{k !} e^{-\lambda} \frac{\beta^{\alpha}}{\Gamma(\alpha)} \lambda^{\alpha-1} e^{-\beta \lambda} d \lambda \\
& =\frac{\beta^{\alpha}}{k ! \Gamma(\alpha)} \int_{0}^{\infty} \lambda^{k+\alpha-1} e^{-(\beta+1) \lambda} d \lambda \\
& =\frac{\beta^{\alpha} \Gamma(k+\alpha)}{(\beta+1)^{k+\alpha} k ! \Gamma(\alpha)} \\
& =\left(\begin{array}{c}
k+\alpha-1 \\
k
\end{array}\right)\left(\frac{1}{\beta+1}\right)^{k}\left(\frac{\beta}{\beta+1}\right)^{\alpha} .
\end{aligned}
$$

In this setting, the distribution of $N$ is the negative binomial distribution with parameter $\left(\alpha, \frac{\beta}{\beta+1}\right)$, usually denoted as $N B\left(\alpha, \frac{\beta}{\beta+1}\right)$. Notice that if we construe $Y_{\lambda}$ as a stochastic process with time parameter $\lambda$ (the so-called Poisson process to be defined later), then $N_{W}$ is the value at time 1 of the process obtained by subordination of $Y_{\lambda}$ by a gamma process. Another way of interpreting this structure is to say that there are various types of customers. Each type will generate its own Poisson random variable with parameter $W$ and the density associated to each customer is given by the law of the random variable $W$, which itself follows the gamma distribution.

If $N \sim N B(n, p)$, then its moment generating function is given by

$$
\begin{aligned}
M_{N}(\theta) & =E\left(e^{\theta N}\right) \\
& =\sum_{k=0}^{\infty} e^{\theta k}\left(\begin{array}{c}
k+n-1 \\
k
\end{array}\right) p^{n}(1-p)^{k} \\
& =\sum_{k=0}^{\infty}\left(\begin{array}{c}
-n \\
k
\end{array}\right) p^{n}(1-p)^{k} e^{\theta k} \\
& =p^{n}\left(1-(1-p) e^{\theta}\right)^{-n} .
\end{aligned}
$$


Makoto Yamazato

Hence the expectation and the variance appear as

$$
E(N)=\frac{n(1-p)}{p}, V(N)=\frac{n(1-p)}{p^{2}},
$$

respectively. Unless $p=1$, we always get $V(N)>E(N)$.

\subsection{The total claim amount}

Consider now the amount of claims. We assume the claim sizes to be positive random variables, mutually independent and identically distributed. We denote them by $X_{1}, X_{2}, \ldots$ Let $S$ be the total amount of claims, so $S=\sum_{k=1}^{N} X_{k}$. Its expectation is

$$
\begin{aligned}
E(S)=E\left(\sum_{k=1}^{N} X_{k}\right) & =E\left(\sum_{k=1}^{n} X_{k} \mid N=n\right) P(N=n) \\
& =E\left(X_{1}\right) \sum_{n=0}^{\infty} n P(N=n) \\
& =E(X) E(N) .
\end{aligned}
$$

Here $X$ is a random variable whose law is identical with the law of all $X_{i}$. The net premium (i.e., the price of the insurance to be paid by each client) is given by estimating $E(N)$ and $E(S)$ from past data and then setting the net premium as $E(X)=E(S) / E(N)$. The above calculation gives $E(S \mid N)=N E(X)$. Let us calculate the variance. Define the conditional variance $V(S \mid N)$ by $V(S \mid N)=E\left(\left(S-E(S \mid N)^{2}\right) \mid N\right)$.

Applying this equality, we obtain

$$
\begin{aligned}
V(S) & =E\left(E\left((S-E(S))^{2} \mid N\right)\right) \\
& =E\left(E\left(((S-E(S \mid N))+(E(S \mid N)-E(S)))^{2} \mid N\right)\right) \\
& =E(V(S \mid N))+V(E(S \mid N)) .
\end{aligned}
$$


Here, we used the fact that $E(S \mid N)-E(S)$ is $\sigma(N)$-measurable. Notice that we did not need $X$ and $N$ to be independent at this stage in order to derive this equality. However, using the independence of $X$ and $N$, we have $V(S \mid N)=N V(X)$ and $E(S \mid N)=N E(X)$. Therefore one gets

$$
V(S)=E(N) V(X)+V(N)(E(X))^{2} .
$$

In the particular case $N \sim \operatorname{Po}(\lambda)$, then $V(N)=E(N)=\lambda$ implies

$$
V(S)=\lambda\left((E(X))^{2}+V(X)\right)=\lambda E\left(X^{2}\right) .
$$

The moment generating function $M_{S}(\theta)=E\left(e^{\theta S}\right)$ of $S$ is given by

$$
\begin{aligned}
M_{S}(\theta) & =E\left(E\left(e^{\theta S} \mid N\right)\right)=E\left(M_{X}(\theta)^{N}\right) \\
& =E\left(\exp \left(N \log M_{X}(\theta)\right)\right)=M_{N}\left(\log M_{X}(\theta)\right) .
\end{aligned}
$$

If $N \sim \operatorname{Po}(\lambda)$, then the moment generating function of $S$ is

$$
\mathrm{M}_{S}(\theta)=\exp \left(\lambda\left(M_{X}(\theta)-1\right)\right),
$$

and the distribution function is given by

$$
F_{S}(x)=P\left(S_{N} \leq x\right)=\sum_{n=0}^{\infty} \frac{\lambda^{n}}{n !} e^{-\lambda} F_{X}^{n *}(x),
$$

where $F_{X}$ is the distribution function of $X$ and $F_{X}^{n *}(n \geq 1)$ is the $n$-fold convolution of $F$ and $F_{X}^{0 *}(x)=\int_{(-\infty, x]} \delta_{0}(d x)$. Here $\delta_{0}(d x)$ denotes the point mass measure at 0 .

The distribution of $S$ discussed previously corresponds to the so called compound Poisson distribution.

If $N \sim N B(n, p)$, we have

$$
E(S)=\frac{n(1-p)}{p} E(X), V(S)=\frac{n(1-p)}{p} V(X)+\frac{n(1-p)}{p^{2}}(E(X))^{2} .
$$

The moment generating function of $S$ is given by

$$
M_{S}(\theta)=p^{n}\left(1-(1-p) M_{X}(\theta)\right)^{-n},
$$


and the distribution function by

$$
F_{S}(x)=\sum_{k=0}^{\infty} F_{X}^{k *}(x)\left(\begin{array}{c}
n+k-1 \\
k
\end{array}\right) p^{n}(1-p)^{k} .
$$

The distribution of $S$ in this case is called the compound negative binomial distribution.

\section{Collective Risk Models}

In the previous chapter, we considered the total claim amount in a unit time interval. In this chapter, we observe the dynamical behavior of the amount of claims. We say that a family of parametrized random variables is a stochastic process regarding the parameter as a time variable. Usually the parameter runs through $[0, \infty)$ or $\{0,1,2, \ldots\}$.

\subsection{Compound Poisson processes}

A stochastic process $\{N(t) ; t \in[0, \infty)\}$ taking values in $\{0,1, \ldots\}$ is called a Poisson process with parameter $\lambda$ if it satisfies the following four conditions.

(1) $N(0)=0$,

(2) for $0 \leq s<t$, the law of $N(t)-N(s)$ is Poisson with parameter $\lambda(t-s)$,

(3) for $0 \leq t_{0}<t_{1}<\cdots<t_{n}$, the random variables $N\left(t_{0}\right), N\left(t_{1}\right)-$ $N\left(t_{0}\right), \ldots, N\left(t_{n}\right)-N\left(t_{n-1}\right)$ are mutually independent, and

(4) $\{N(t) ; t \in[0, \infty)\}$ is right continuous with left limits.

Construction of a Poisson process. Let $T_{1}, T_{2}, \ldots$ be a sequence of independent random variables with common exponential distribution with parameter $\lambda>0$ and let $S_{0}=0, S_{n}=T_{1}+\cdots+T_{n}(n \geq 1)$. Define $N_{t}=N(t)$ by

$$
N_{t}=N(t)=n, \quad \text { if } S_{n} \leq t<S_{n+1} .
$$


Obviously we have $N_{0}=0$, and $N_{t}$ is by definition right continuous with left limits. Let us prove conditions (2) and (3) simultaneously. For $0 \leq t_{1}<t_{2}<\cdots<t_{n}$ and $0 \leq k_{1} \leq \cdots \leq k_{n}$, we have

$$
\begin{aligned}
& P\left(N_{t_{1}}=k_{1}, N_{t_{2}}-N_{t_{1}}=k_{2}-k_{1}, \ldots, N_{t_{n}}-N_{t_{n-1}}=k_{n}-k_{n-1}\right) \\
= & P\left(N_{t_{1}}=k_{1}, N_{t_{2}}=k_{2}, \ldots, N_{t_{n}}=k_{n}\right) \\
= & P\left(S_{k_{1}} \leq t_{1}<S_{k_{1}+1}, \cdots, S_{k_{n}} \leq t_{n}<S_{k_{n}+1}\right) \\
= & \int \cdots \int_{D_{n} \cap\left\{t_{n}<s_{k_{n}}+u_{k_{n}+1}\right\}} \lambda^{k_{n}+1} e^{-\lambda\left(s_{k_{n}}+u_{k_{n}+1}\right)} d u_{1} \cdots d u_{k_{n}+1} \\
= & I_{n},
\end{aligned}
$$

where $s_{k}=u_{1}+\cdots+u_{k}$, for $k=1,2, \ldots$, and

$$
D_{n}=\left\{s_{1} \leq \cdots \leq s_{k_{1}} \leq t_{1}<s_{k_{1}+1} \leq \cdots \leq s_{k_{n}} \leq t_{n}\right\} \subset \mathbb{R}^{k_{n}+1} .
$$

Integrating (1) with respect to $u_{k_{n}+1}$ on $\left[t_{n}-s_{k_{n}}, \infty\right)$, we obtain $I_{n}=$ $\lambda^{k_{n}} e^{-\lambda t_{n}} \int_{D_{n}} d s_{1} \cdots d s_{k_{n}}$. Set

$$
E_{\ell}=\left\{t_{\ell-1}<s_{k_{\ell-1}+1} \leq \cdots \leq s_{k_{\ell}} \leq t_{\ell}\right\}
$$

for $\ell=1,2, \ldots, n$. Then we get $D_{n}=E_{1} \times \cdots \times E_{n}$, and so

$$
\int_{E_{\ell}} d s_{k_{\ell-1}} \cdots d s_{k_{\ell}}=\frac{\left(t_{\ell}-t_{\ell-1}\right)^{k_{\ell}-k_{\ell-1}}}{\left(k_{\ell}-k_{\ell-1}\right) !}
$$

Hence we have

$$
I_{n}=\frac{\left(\lambda t_{1}\right)^{k_{1}}}{k_{1} !} e^{-\lambda t_{1}} \prod_{i=2}^{n} \frac{\left\{\lambda\left(t_{i}-t_{i-1}\right)\right\}^{k_{i}-k_{i-1}}}{\left(k_{i}-k_{i-1}\right) !} e^{-\lambda\left(t_{i}-t_{i-1}\right)} .
$$

Letting $n=2$ we obtain

$$
\begin{aligned}
P\left(N_{t}-N_{s}=k\right) & =\sum_{j=0}^{\infty} P\left(N_{s}=j, N_{t}-N_{s}=k\right) \\
& =\sum_{j=0}^{\infty} \frac{(\lambda s)^{j}}{j !} e^{-\lambda s} \frac{\{\lambda(t-s)\}^{k}}{k !} e^{-\lambda(t-s)} \\
& =\frac{\{\lambda(t-s)\}^{k}}{k !} e^{-\lambda(t-s)} .
\end{aligned}
$$

Pro Mathematica, 28, 55 (2014), 85-127 
Plugging this last relation into the first one we conclude the equality

$$
\begin{aligned}
& P\left(N_{t_{1}}=k_{1}, N_{t_{2}}-N_{t_{1}}=k_{2}-k_{1}, \ldots, N_{t_{n}}-N_{t_{n-1}}=k_{n}-k_{n-1}\right) \\
= & P\left(N_{t_{1}}=k_{1}\right) \prod_{i=2}^{n} P\left(N_{t_{i}}-N_{t_{i-1}}=k_{i}-k_{i-1}\right) .
\end{aligned}
$$

Next we define what a Lévy process is. A stochastic process $\left\{X_{t} ; t \in\right.$ $[0, \infty)\}$ on $\mathbb{R}^{d}$ is called a Lévy process if it satisfies the following four conditions.

(1) $X_{0}=0$,

(2) for any $n \geq 1$ and $0 \leq t_{0}<t_{1}<\cdots<t_{n}$, the random variables $X_{t_{0}}, X_{t_{1}}-X_{t_{0}}, \ldots, X_{t_{n}}-X_{t_{n-1}}$ are mutually independent,

(3) for $0 \leq s<t$, the distribution of $X_{t}-X_{s}$ is identical to the distribution of $X_{t-s}$, and

(4) $\left\{X_{t} ; t \in[0, \infty)\right\}$ is right continuous with left limits.

In the sequel $(a \wedge b)$ represents the minimum between $a, b \in \mathbb{R}$.

Theorem 3.1. If $\left\{X_{t}\right\}$ is a Lévy process on $\mathbb{R}^{d}$, then its characteristic function $E\left(e^{i<\theta, X_{t}>}\right)$, with $\theta \in \mathbb{R}^{d}$, is given by

$$
\exp \left[t\left\{i\langle\theta, \gamma\rangle-\frac{|\sigma \theta|^{2}}{2}+\int_{\mathbb{R}^{d} \backslash\{0\}}\left(e^{i<\theta, x>}-1-i\langle\theta, x\rangle 1_{\{|x| \leq 1\}}\right) \nu(d x)\right\}\right],
$$

here $\gamma$ is in $\mathbb{R}^{d}$ and $\sigma$ is a $d \times d$-symmetric nonnegative definite matrix, while $\nu$ is a nonnegative measure on $\mathbb{R}^{d} \backslash\{0\}$ satisfying $\int\left(|x|^{2} \wedge 1\right) \nu(d x)<$ $\infty$.

Proof. We refer to Sato [7] for the proof.

A Lévy process $\left\{X_{t}\right\}$ on $\mathbb{R}^{d}$ is called a compound Poisson process if its characteristic function is represented as

$$
\exp \left\{t \int_{\mathbb{R}^{d} \backslash\{0\}} e^{i<\theta, x>}-1 \nu(d x)\right\} .
$$


Here $\nu(d x)$ is a measure on $\mathbb{R}^{d} \backslash\{0\}$ that satisfies $\int_{\mathbb{R}^{d} \backslash\{0\}} \nu(d x)<\infty$.

Construction of a compound Poisson processes. Let $F$ be a probability measure on $\mathbb{R}^{d} \backslash\{0\}$ and $X_{1}, X_{2}, \cdots$ be a sequence of $\mathbb{R}^{d}$-valued independent random variables with common distribution $F$. Let $\left\{N_{t}\right\}$ be a Poisson process with parameter $\lambda>0$, independent from $X_{1}, X_{2}, \cdots$. Then, the stochastic process defined by

$$
Y_{t}= \begin{cases}0 & \text { if } N_{t}=0 \\ X_{1}+\cdots+X_{N_{t}} & \text { otherwise }\end{cases}
$$

is a compound Poisson process with $\nu(d x)=\lambda F(d x)$.

Let us proof this fact. For times $0=t_{0} \leq t_{1}<t_{2} \ldots<t_{n}$ and sets $B_{1}, B_{2}, \ldots, B_{n} \in \mathcal{B}\left(\mathbb{R}^{d}\right)$ we have (the sums are always taken along all possible $\left.0=m_{0} \leq m_{1} \leq m_{2} \leq \cdots \leq m_{n}\right)$

$$
\begin{aligned}
& P\left(Y_{t_{1}} \in B_{1}, Y_{t_{2}}-Y_{t_{1}} \in B_{2}, \ldots, Y_{t_{n}}-Y_{t_{n-1}} \in B_{n}\right)= \\
& =\sum \prod_{i=1}^{n} P\left(X_{m_{i-1}+1}+\cdots+X_{m_{i}} \in B_{i}\right) P\left(N_{t_{i}}-N_{t_{i-1}}=m_{i}-m_{i-1}\right) \\
& =\sum \prod_{i=1}^{n} P\left(X_{1}+\cdots+X_{m_{i}-m_{i-1}} \in B_{i}\right) P\left(N_{t_{i}-t_{i-1}}=m_{i}-m_{i-1}\right) \\
& =\sum \prod_{i=1}^{n} P\left(Y_{m_{i}-m_{i-1}} \in B_{i}, N_{t_{i}-t_{i-1}}=m_{i}-m_{i-1}\right) \\
& =\prod_{i=1}^{n} P\left(Y_{t_{i}-t_{i-1}} \in B_{i}\right) .
\end{aligned}
$$

On the other hand, if $t \geq s$, from

$$
\begin{aligned}
P\left(Y_{t}-Y_{s} \in B\right) & =\sum_{0 \leq m \leq n} P\left(X_{m+1}+\cdots+X_{n} \in B, N_{s}=m, N_{t}=n\right) \\
& =\sum_{0 \leq m \leq n} P\left(X_{1}+\cdots+X_{n-m} \in B, N_{s}=m, N_{t}=n\right)
\end{aligned}
$$

Pro Mathematica, 28, 55 (2014), 85-127 
Makoto Yamazato

$$
\begin{aligned}
& =\sum_{0 \leq m \leq n} P\left(X_{1}+\cdots+X_{n-m} \in\right. \\
& \left.\quad B, N_{t-s}=n-m\right) \times \\
& \quad \times\left(N_{s}=m\right) \\
& =\sum_{0 \leq m \leq n} P\left(Y_{t-s} \in B, N_{t-s}=n-m\right) P\left(N_{s}=m\right) \\
& =P\left(Y_{t-s} \in B\right)
\end{aligned}
$$

we get $Y_{t}-Y_{s} \sim Y_{t-s}$.

Also, by the very definition of $Y$ we get

$$
\begin{aligned}
P\left(Y_{t} \in A\right) & =\delta_{0}(A)+\sum_{n \geq 1} P\left(X_{1}+\cdots+X_{n} \in A\right) P(N(t)=n) \\
& =\sum_{n \geq 0} F^{n *}(A) \frac{(\lambda t)^{n}}{n !} e^{-\lambda t} .
\end{aligned}
$$

Hence we obtain

$$
\begin{aligned}
E\left(e^{i<\theta, Y_{t}>}\right) & =\sum_{n \geq 0} \int e^{i<\theta, y>} F^{n *}(d y) \frac{(\lambda t)^{n}}{n !} e^{-\lambda t} \\
& =\sum_{n \geq 0}\left(\int e^{i<\theta, y>} F(d y)\right)^{n} \frac{(\lambda t)^{n}}{n !} e^{-\lambda t} \\
& =\exp \left\{t \int_{R^{d} \backslash\{0\}}\left(e^{i<\theta, x>}-1\right) \nu(d x)\right\} .
\end{aligned}
$$

\subsection{Risk processes}

Let $Y_{t}$ be a compound Poisson process on $[0, \infty)$ with characteristic function

$$
E\left(e^{i \theta Y_{t}}\right)=\exp \left\{\lambda t \int_{(0, \infty)}\left(e^{i \theta x}-1\right) F(d x)\right\}
$$

We call $R(t)=c t-Y_{t}$ the risk process and $U(t)=u+R(t)$ the surplus reserve process. We assume here that the expectation $\mu=$ 
$\int_{(0, \infty)} x F(d x)=\int_{0}^{\infty} 1-F(x) d x$ of $F$ is finite. We call the parameter $\rho=\frac{c}{\lambda \mu}-1$ the safety loading. We always assume $\rho>0$.

Let $\tau_{u}$ be the hitting time of $(-\infty, 0)$ for $U(t)$; that is,

$$
\tau_{u}=\inf \{t \geq 0 ; U(t) \in(-\infty, 0)\} .
$$

This hitting time is called ruin time and $\mathcal{E}(u)=P\left(\tau_{u}<\infty\right)$ the ruin probability in insurance mathematics. Here $c$ represents the rate of premiums that the insurer receives per unit of time and $u$ is the initial capital of the insurance company.

Lemma 3.2. For $A, B, C \in \mathcal{B}(\mathbb{R})$, we have

$$
\begin{aligned}
& P\left(R\left(T_{1}\right) \in A, R\left(T_{1}+t\right) \in B, T_{1} \in C\right)= \\
& \quad \int_{C} \int_{A} P(z+R(t) \in B) P\left(c s-X_{1} \in d z\right) P\left(T_{1} \in d s\right) .
\end{aligned}
$$

Proof. For $A, B, C \in \mathcal{B}(\mathbb{R})$, we have

$$
\begin{aligned}
& P\left(R\left(T_{1}\right) \in A, R\left(T_{1}+t\right) \in B, T_{1} \in C\right)= \\
= & \sum_{m=1}^{\infty} \int_{C} P\left(c s-X_{1} \in A, N_{s+t}-N_{s}=m-1, R\left(T_{1}+t\right) \in B \mid T_{1}=s\right) \\
\times & P\left(T_{1} \in d s\right) \\
= & \sum_{m=1}^{\infty} \int_{C} P\left(c s-X_{1} \in A, N_{s}=1, N_{s+t}-N_{s}=m-1,\right. \\
\left.c(s+t)-\left(X_{1}+\cdots+X_{m}\right) \in B \mid T_{1}=s\right) P\left(T_{1} \in d s\right) & \\
= & \sum_{m=1}^{\infty} \int_{C} P\left(c t-X_{1} \in A, T_{2}+\cdots+T_{m} \leq t<T_{2}+\cdots+T_{m+1},\right. \\
c & \left.\sum_{m=1}^{\infty} \int_{C} P(s+t)-\left(X_{1}+\cdots+X_{m}\right) \in B \mid T_{1}=s\right) P\left(T_{1} \in d s\right) \\
& \left.X_{1} \in A, c(s+t)-\left(X_{1}+\cdots+X_{m}\right) \in B\right) \\
\times & P\left(T_{2}+\cdots+T_{m} \leq t<T_{2}+\cdots+T_{m+1}\right) P\left(T_{1} \in d s\right)
\end{aligned}
$$




$$
\begin{aligned}
= & \sum_{m=1}^{\infty} P\left(N_{t}=m-1\right) \times \\
& \quad \times \int_{C} P\left(c s-X_{1} \in A, c s-X_{1}+c t-\left(X_{2} \cdots+X_{m}\right) \in B\right) P\left(T_{1} \in d s\right) \\
= & \sum_{m=1}^{\infty} P\left(N_{t}=m-1\right) \times \\
& \quad \times \int_{C} \int_{A} P\left(z+c t-\left(X_{2} \cdots+X_{m}\right) \in B\right) P\left(c s-X_{1} \in d z\right) P\left(T_{1} \in d s\right) \\
= & \int_{C} \int_{A} P(z+R(t) \in B) P\left(c s-X_{1} \in d z\right) P\left(T_{1} \in d s\right) .
\end{aligned}
$$

The following theorem exhibits a closed formula for the ruin probability based on the claim distribution.

Theorem 3.3. Let $h(y)=\frac{1-F(y)}{\mu}$ and $H(x)=\int_{0}^{x} h(y) d y$. Then $H$ is a distribution function and the following equality

$$
\mathcal{E}(u)=1-\frac{\rho}{1+\rho} \sum_{n=0}^{\infty} \frac{H^{n *}(u)}{(1+\rho)^{n}}
$$

holds.

Proof. Let $\varphi(u)=1-\mathcal{E}(u)$ be the chance of never get ruined. As the ruin can never happen before the first event, we have

$$
\begin{aligned}
\varphi(u)= & P(U(t) \geq 0, \text { for all } t \geq 0) \\
= & P\left(u+R\left(T_{1}\right) \geq 0, u+R\left(T_{1}+t\right) \geq 0, \text { for all } t \geq 0\right) \\
= & \int_{0}^{\infty} \int_{(0, u+c s]} P(u+c s-z+R(t) \geq 0, \text { for all } t \geq 0) \times \\
& \times P\left(X_{1} \in d z\right) P\left(T_{1} \in d s\right) \\
= & \int_{0}^{\infty} \lambda e^{-\lambda s}\left\{\int_{(0, u+c s]} \varphi(u+c s-z) F(d z)\right\} d s .
\end{aligned}
$$


With the change of variable $t=u+c s$, we arrive at

$$
\varphi(u)=\frac{\lambda}{c} e^{\lambda u / c} \int_{u}^{\infty} e^{-\lambda t / c}\left\{\int_{(0, t]} \varphi(t-z) F(d z)\right\} d t .
$$

Hence $\varphi(u)$ is continuous, right differentiable, and satisfies

$$
\varphi^{\prime}(u)=\frac{\lambda}{c} \varphi(u)-\frac{\lambda}{c}\left\{\int_{(0, u]} \varphi(u-z) F(d z)\right\} .
$$

Noting that by Fubini's theorem we have

$$
\begin{aligned}
\int_{0}^{u}\left(\int_{(0, v]} \varphi(v-x) F(d x)\right) d v & =\int_{(0, u]}\left(\int_{0}^{u-x} \varphi(v) d v\right) F(d x) \\
& =\int_{0}^{u} \varphi(v)\left(\int_{(0, u-v]} F(d x)\right) d v \\
& =\int_{0}^{u} \varphi(u-v) F(v) d v
\end{aligned}
$$

one then integrates (2) to obtain

$$
\begin{aligned}
\varphi(u) & =\varphi(0)+\frac{\lambda}{c}\left(\int_{0}^{u} \varphi(v) d v-\int_{0}^{u}\left(\int_{(0, v]} \varphi(v-x) F(d x)\right) d v\right) \\
& =\varphi(0)+\frac{\lambda}{c} \int_{0}^{u} \varphi(u-z)[1-F(z)] d z \\
& =\varphi(0)+\frac{1}{1+\rho} \int_{0}^{u} \varphi(u-z) H(d z) .
\end{aligned}
$$

Letting $u \rightarrow \infty$, by monotone convergence we get

$$
\varphi(\infty)=\varphi(0)+\frac{\lambda \mu}{c} \varphi(\infty)
$$

where $\mu=\int_{0}^{\infty} 1-F(z) d z$.

On the other hand, let

$$
\Omega_{0}=\left\{\omega \in \Omega: \lim _{t \rightarrow \infty} \frac{R_{t}(\omega)}{t}=c-\lambda \mu\right\} .
$$

Pro Mathematica, 28, 55 (2014), 85-127 
Then the strong law of large numbers implies $\lim _{t \rightarrow \infty} \frac{N_{t}(\omega)}{t}=\lambda>0$ and $\lim _{t \rightarrow \infty} \frac{1}{N_{t}(\omega)} \sum_{i=1}^{N_{t}(\omega)} X_{i}(\omega)=\mu$ for almost all $\omega$. Therefore $P\left(\Omega_{0}\right)=1$.

Since $c>\lambda \mu$, for $\omega \in \Omega_{0}$, there is $u>0$ such that for all $t \geq 0$ we have $R_{t}(\omega)>-u$. Hence we get

$$
\begin{aligned}
\varphi(\infty) & =\lim _{u \rightarrow \infty} \varphi(u) \\
& =\lim _{u \rightarrow \infty} P\left(\cap_{t \geq 0}\left\{R_{t}>-u\right\}\right) \\
& =\lim _{n \rightarrow \infty} P\left(\cap_{t \in \mathbb{Q}_{+}}\left\{R_{t}>-n\right\}\right) \\
& =P\left(\cup_{n \geq 1} \cap_{t \in \mathbb{Q}_{+}}\left\{R_{t}>-n\right\}\right)=1 .
\end{aligned}
$$

By this equality and (5), we have

$$
\varphi(0)=\frac{\rho}{1+\rho} \quad \text { and } \quad \mathcal{E}(0)=\frac{1}{1+\rho} .
$$

Set $\widehat{H}(\theta)=\int_{0}^{\infty} e^{-\theta x} h(x) d x$ and $\widehat{\varphi}(\theta)=\int_{[0, \infty)} e^{-\theta x} \varphi(d x)$. Then by $(4)$ we get

$$
\widehat{\varphi}(\theta)=\frac{\rho}{1+\rho}+\frac{1}{1+\rho} \widehat{\varphi}(\theta) \widehat{H}(\theta) .
$$

So at the end we conclude

$$
\widehat{\varphi}(\theta)=\frac{\frac{\rho}{1+\rho}}{1-\frac{1}{1+\rho} \widehat{H}(\theta)} .
$$

Example 3.4. Let $F(x)=1-e^{-x / \mu}$. Then $H(x)=1-e^{-x / \mu}$ and hence by the above theorem, $\mathcal{E}(x)=\frac{1}{1+\rho} e^{-\rho x /(1+\rho) \mu}$.

Remark 3.5. Let $Y_{1}, Y_{2}, \ldots$ be a sequence of independent random variables with distribution function $H$, and $M$ be a random variable independent of $Y_{1}, Y_{2}, \ldots$ which have geometric distribution with parameter $\rho(1+\rho)^{-1}$, that is,

$$
P(M=n)=\frac{\rho}{1+\rho}(1+\rho)^{-n}, n=0,1, \cdots .
$$


Then we have

$$
\mathcal{E}(u)=P\left(Y_{1}+Y_{2}+\cdots+Y_{M}>u\right) .
$$

Theorem 3.6. For $G(u, y)=P\left(U\left(\tau_{u}\right) \in[-y, 0), \tau_{u}<\infty\right)$ we have $G(0, y)=\frac{\lambda}{c} \int_{0}^{y}(1-F(u) d u$.

Proof. In the case of our concern, we must have $\tau_{u}=\sum_{k=1}^{m} T_{k}$ for some $m \geq 1$. As in Lemma 3.2, we have then

$$
\begin{aligned}
G(u, y)= & P\left(U\left(T_{1}\right) \in[-y, 0)\right) \\
+\sum_{m=2}^{\infty} P( & \left.U\left(T_{1}\right) \geq 0, \ldots, U\left(\sum_{k=1}^{m-1} T_{k}\right) \geq 0, U\left(\sum_{k=1}^{m} T_{k}\right) \in[-y, 0)\right) \\
= & \iint_{-y \leq u+c t-x<0} \lambda e^{-\lambda t} F(d x) d t \\
& +\iint_{0 \leq u+c t-x<u+c t} G(u+c t-x, y) \lambda e^{-\lambda t} F(d x) d t .
\end{aligned}
$$

However, the first integral equals

$$
\begin{aligned}
& =\int_{0}^{\infty}\{F(u+c t+y)-F(u+c t)\} \lambda e^{-\lambda t} d t \\
& =\int_{u}^{\infty}\{F(s+y)-F(s)\} \frac{\lambda}{c} e^{-\lambda(s-u) / c} d s \\
& =\frac{\lambda}{c} e^{\lambda u / c} \int_{u}^{\infty}\{F(s+y)-F(s)\} e^{-\lambda s / c} d s,
\end{aligned}
$$

while the second is

$$
\begin{aligned}
& =\int_{0}^{\infty}\left(\int_{(0, u+c t]} G(u+c t-x, y) F(d x)\right) \lambda e^{-\lambda t} d t \\
& =\int_{u}^{\infty}\left(\int_{(0, s]} G(s-x, y) F(d x)\right) \frac{\lambda}{c} e^{-\lambda(s-u) / c} d s .
\end{aligned}
$$

Pro Mathematica, 28, 55 (2014), 85-127 
Makoto Yamazato

From the above calculations we conclude that $G$ is right differentiable in $u$ and satisfies

$\frac{\partial}{\partial u} G(u, y)=\frac{\lambda}{c}\left[G(u, y)-\{F(u+y)-F(u)\}-\int_{(0, u]} G(u-x, y) F(d x)\right]$.

Integrating from 0 to $M$ with respect to $u$, we have

$$
\begin{aligned}
G(M, y)-G(0, y)= & -\frac{\lambda}{c}\left[\int_{0}^{M}\{F(u+y)-F(u)\} d u\right. \\
& \left.+\int_{0}^{M}\left\{G(u, y)-\int_{(0, u]} G(u-x, y) F(d x)\right\} d u\right]
\end{aligned}
$$

By (3) we get

$$
\begin{aligned}
& \int_{0}^{M}\left\{G(u, y)-\int_{0}^{u} G(u-x, y) F(d x)\right\} d u= \\
& \quad=\int_{0}^{M} G(M-x, y)(1-F(x)) d x \rightarrow 0 \quad(M \rightarrow \infty) .
\end{aligned}
$$

Here we have applied the equality $G(\infty, y)=0$ and the Lebesgue's dominated convergence theorem. Hence we conclude

$$
\begin{aligned}
-G(0, y) & =G(\infty, y)-G(0, y) \\
& =-\frac{\lambda}{c} \int_{0}^{\infty}\{F(u+y)-F(u)\} d u \\
& =-\frac{\lambda}{c} \int_{0}^{\infty}\{(F(u+y)-1)+(1-F(u))\} d u \\
& =-\frac{\lambda}{c} \int_{0}^{y}(1-F(u)) d u .
\end{aligned}
$$

Let $F, G$ be two distribution functions. The number $L(F, G)=$ $\inf \{h>0: F(x-h)-h \leq G(x) \leq F(x+h)+h$, for all $x \in \mathbb{R}\}$ is called the Lévy's distance from $F$ to $G$. 
Problem 3.7. Show that $L$ satisfies the axioms of distance in a metric space.

Lemma 3.8. Let $F_{n}, F$ be distribution functions. For every continuity point of $F$ we have $\lim F_{n}(x)=F(x)$ if and only if $\lim L\left(F_{n}, F\right)=0$.

Proof. We first deal with the reverse implication. If $h \geq L\left(F_{n}, F\right)$, then for each $x$ we have

$$
F(x-h)-h \leq F_{n}(x) \leq F(x+h)+h .
$$

As the inequality

$$
F(x-h)-h \leq F(x) \leq F(x+h)+h
$$

always holds, we get $\left|F(x)-F_{n}(x)\right| \leq F(x+h)-F(x-h)+2 h$. Hence, if $x$ is a continuity point of $F(x)$, we have the $\operatorname{limit}_{\lim _{n \rightarrow \infty}} F_{n}(x)=F(x)$.

Conversely, choose $0<\epsilon<1$ arbitrary. As any increasing right continuous function has at most a countable number of discontinuities, we can choose continuity points $x_{0}<x_{1}<\ldots, x_{k}$ of $F$ so that $F\left(x_{0}\right)<\epsilon$, $F\left(x_{k}\right)>1-\epsilon$, and $x_{i}-x_{i-1}<\epsilon, i=1, \ldots, k$. Choose $n_{0}$ so that $\left|F\left(x_{i}\right)-F_{n}\left(x_{i}\right)\right|<\epsilon$ for all $0 \leq i \leq k$ and $n \geq n_{0}$. Then for $x$ with $x_{i-1}<x<x_{i}$ we get

$$
\begin{aligned}
F_{n}(x-\epsilon)-\epsilon \leq F_{n}\left(x_{i-1}\right)-\epsilon & <F\left(x_{i-1}\right) \\
& \leq F(x) \\
& \leq F\left(x_{i}\right) \leq F_{n}\left(x_{i}\right)+\epsilon \leq F_{n}(x+\epsilon)+\epsilon,
\end{aligned}
$$

and for $x<x_{0}$,

$F_{n}(x-2 \epsilon)-2 \epsilon \leq F_{n}\left(x_{0}\right)-\epsilon-F\left(x_{0}\right) \leq 0 \leq F(x)<\epsilon \leq \epsilon+F_{n}(x+\epsilon)$.

For $x>x_{k}$, we have the similar estimate, thus we obtain $L\left(F_{n}, F\right) \leq$ $2 \epsilon$.

Lemma 3.9. Let $F, G$ be distribution functions. If $G$ has a bounded density $g$, then we have

$$
\sup _{x}|F(x)-G(x)| \leq\left(1+\sup _{x} g(x)\right) L(F, G) .
$$


Proof. Set $g^{*}=\sup _{x} g(x)$. Let $h>0$ be so that $G(x-h)-h \leq F(x) \leq$ $G(x+h)+h$ holds for any $x>0$. Then as we have

$G(x)-h\left(g^{*}+1\right) \leq G(x-h)-h \leq F(x) \leq G(x+h)+h \leq G(x)+h\left(g^{*}+1\right)$,

we obtain $|F(x)-G(x)| \leq h\left(1+g^{*}\right)$. Finally, take the supremum with respect to $x$ and then the infimum with respect to $h$ in order to stablish the result.

Theorem 3.10 (Rényi). Let $Y_{1}, Y_{2}, \ldots$ be a sequence of nonnegative independent and identically distributed random variables. Let $M_{\epsilon}$ be a geometric random variable with parameter $\epsilon$ independent of $\left\{Y_{n}\right\}_{n \geq 1}$. Set $S_{\epsilon}=Y_{1}+\cdots+Y_{M_{\epsilon}}$ and let $\alpha=E Y_{1}$ and $F_{\epsilon}(x)=P\left(\epsilon \alpha^{-1} S_{\epsilon} \leq x\right)$. We have then

$$
\lim _{\epsilon \rightarrow 0} \sup _{x \geq 0}\left|F_{\epsilon}(x)-1+e^{-x}\right|=0 .
$$

Proof. Conditioning with respect to $M_{\epsilon}$ and using the independent and identically distributed property of the sequence $Y_{i}$, one obtains

$$
\begin{aligned}
E\left(\exp \left(i \theta \epsilon \alpha^{-1} S_{\epsilon}\right)\right) & =\sum_{n=0}^{\infty} E\left[\exp \left\{i \theta \epsilon \alpha^{-1}\left(Y_{1}+\cdots+Y_{n}\right)\right\}\right] P\left(M_{\epsilon}=n\right) \\
& =\sum_{n=0}^{\infty}\left(E \exp \left(i \theta \epsilon \alpha^{-1} Y_{1}\right)\right)^{n} \epsilon(1-\epsilon)^{n} \\
& =\frac{\epsilon}{1-(1-\epsilon) E \exp \left(i \theta \epsilon \alpha^{-1} Y_{1}\right)} \rightarrow \frac{1}{1-i \theta}(\epsilon \rightarrow 0) .
\end{aligned}
$$

Note that for the last equality we have used L'Hopital's rule. Then we have

$$
\lim _{\epsilon \rightarrow 0}\left|F_{\epsilon}(x)-1+e^{-x}\right|=0
$$

for all $x \geq 0$ by Lemma 3.8 (see also Lemma 4.17). Finally, by Lemmas 3.8 and 3.9 , one obtains the claimed result. 
Theorem 3.11. If $E\left(X_{1}^{2}\right)<\infty$, then we have

$$
\lim _{\rho \rightarrow 0} \sup _{u \geq 0}\left|\mathcal{E}(u)-\exp \left\{\frac{-2 \rho \mu u}{(1+\rho) E\left(X_{1}^{2}\right)}\right\}\right|=0 .
$$

Proof. Let $Y_{1}, Y_{2}, \cdots$ be nonnegative independent identically distributed random variables with density function $\frac{1-F(x)}{\mu}$ and let $M$ be a geometric random variable with parameter $\frac{\rho}{1+\rho}$ independent of $Y_{1}, Y_{2}, \ldots$, that is, that behave like

$$
P(M=n)=\frac{\rho}{1+\rho}(1+\rho)^{-n}, n=0,1, \cdots .
$$

By Remark 3.5, we have then

$$
\mathcal{E}(u)=P\left(Y_{1}+\cdots+Y_{M}>u\right) .
$$

Set $\epsilon=\frac{\rho}{1+\rho}, S_{\epsilon}=Y_{1}+\cdots+Y_{M}$ and $x=\frac{\rho}{1+\rho} \frac{u}{E\left(Y_{1}\right)}$, and then apply Rényi's theorem. So we have

$$
\begin{aligned}
& \lim _{\rho \rightarrow 0} \sup _{u \geq 0}\left|\mathcal{E}(u)-\exp \left\{\frac{-\rho u}{(1+\rho) E Y_{1}}\right\}\right| \\
= & \lim _{\epsilon \rightarrow 0} \sup _{x}\left|P\left(\frac{\epsilon S_{\epsilon}}{E\left(Y_{1}\right)}>x\right)-e^{-x}\right|=0 .
\end{aligned}
$$

Due to integration by parts we have $E\left(Y_{1}\right)=\mu^{-1} \int_{0}^{\infty} y(1-F(y)) d y=$ $\frac{1}{2 \mu} E\left(X_{1}^{2}\right)$. The conclusion follows.

Example 3.12. If the distribution of the random variable $X_{1}$ is an exponential distribution with mean $\mu$, then because of $E\left(X_{1}^{2}\right)=2 \mu^{2}$ and Example 3.4 we obtain

$$
\left|\mathcal{E}(u)-\exp \left\{\frac{-2 \rho \mu u}{(1+\rho) E\left(X_{1}^{2}\right)}\right\}\right|=\frac{\rho}{1+\rho} \exp \left\{\frac{-\rho u}{(1+\rho) \mu}\right\} .
$$

Hence we get

$$
\lim _{\rho \rightarrow 0} \sup _{u \geq 0}\left|\mathcal{E}(u)-\exp \left\{\frac{-2 \rho \mu u}{(1+\rho) E\left(X_{1}^{2}\right)}\right\}\right|=\lim _{\rho \rightarrow 0} \frac{\rho}{1+\rho}=0,
$$

and the asymptotic behavior of ruin probability as $\rho \rightarrow 0$ is compatible with the conclusion of Theorem 3.11. 


\section{Asymptotics of Ruin Probabilities (light tail claims)}

In the rest of this article we will describe the asymptotic behavior of the ruin probability as the initial surplus $u$ tends to infinity. First we will discuss the Cramér-Lundberg approximation which is treated in standard actuarial textbooks. Roughly speaking, this is related to an exponential decay of the tail of the claim distribution.

\subsection{Cramér-Lundberg approximation}

A distribution function $F(x)$ satisfies the Cramér-Lundberg condition if there is an $r^{*}>0$ such that

$$
\frac{\lambda}{c} \int_{0}^{\infty} e^{r^{*} x}[1-F(x)] d x=1 .
$$

The parameter $r^{*}$ is called the Cramér-Lundberg exponent or adjustment coefficient. In actuarial textbooks this exponent is usually denoted by $R$. Here, in order to avoid confusion, we use $r^{*}$. The above equation is rewritten using the integration by parts formula as

$$
\int_{[0, \infty)} e^{r^{*} x} F(d x)=1+\frac{c r^{*}}{\lambda} .
$$

Theorem 4.1. We have

$$
\mathcal{E}(u) \leq E\left(\exp \left[-r^{*} U\left(\tau_{u}\right)\right]: \tau_{u}<\infty\right)=e^{-r^{*} u} .
$$

Proof. Since $U\left(\tau_{u}\right) \leq 0$, the first inequality is obvious. Define

$$
Z(t)=\exp \left[-r^{*}\{R(t)+u\}\right]
$$

and $\mathcal{F}_{t}=\sigma\{Z(s) ; s \leq t\}$. Then $Z(t)$ is an $\left\{\mathcal{F}_{t}\right\}$-martingale. In fact, by the Cramér-Lundberg condition and the independent increment property 
of the risk process, we have

$$
\begin{aligned}
E\left(Z(t+s) \mid \mathcal{F}_{t}\right) & =e^{-r^{*}[R(t)+u]} E\left(e^{-r^{*} R(s)}\right) \\
& =Z(t) \exp \left[\lambda s\left(E\left(e^{r^{*} X_{1}}\right)-1\right)\right] e^{-r^{*} c s} \\
& =Z(t) .
\end{aligned}
$$

Therefore by the optional sampling theorem, we obtain

$$
E\left(Z\left(\tau_{u} \wedge t\right) \mid \mathcal{F}_{0}\right)=Z(0)=e^{-r^{*} u} .
$$

On the other hand,

$$
\begin{aligned}
E\left(Z\left(\tau_{u} \wedge t\right) \mid \mathcal{F}_{0}\right) & =E\left(Z\left(\tau_{u} \wedge t\right)\right) \\
& =E\left(Z\left(\tau_{u}\right): \tau_{u} \leq t\right)+E\left(Z(t): \tau_{u}>t\right)
\end{aligned}
$$

holds. The first term of the right hand side of the above equality tends to $E\left(Z\left(\tau_{u}\right): \tau_{u}<\infty\right)$ as $t \rightarrow \infty$. By the strong law of large numbers we get $\lim _{t \rightarrow \infty} Z(t)=0$ a.s. and $R(t, \omega)+u \geq 0$ for $\omega \in\left\{\tau_{u}(\omega)>t\right\}$; hence $0 \leq Z(t) 1_{\left\{\tau_{u}>t\right\}} \leq 1$. Now, by the bounded convergence theorem, we get

$$
\begin{aligned}
\lim _{t \rightarrow \infty} E\left(Z(t) 1_{\left\{\tau_{u}>t\right\}}\right) & =E\left(\lim _{t \rightarrow \infty} Z(t) 1_{\left\{\tau_{u}>t\right\}}\right) \\
& =0 .
\end{aligned}
$$

Therefore, we must have

$$
E\left(Z\left(\tau_{u}\right): \tau_{u}<\infty\right)=e^{-r^{*} u}
$$

Theorem 4.2 (The law of large numbers). If $R(t)$ is the risk process, then we have

$$
P\left(\lim _{t \rightarrow \infty} \frac{R(t)}{t}=E(R(1))\right)=1
$$


Proof. Note that $R(n)=\sum_{i=0}^{n-1}(R(i+1)-R(i)), n=1,2, \ldots$, are the sums of independent and identically distributed random variables and $E(R(n))=n E(R(1))$ is finite. By the usual strong law of large numbers, we can thus conclude

$$
P\left(\lim _{n \rightarrow \infty} \frac{R(n)}{n}=E(R(1))\right)=1 .
$$

Set $V_{n}=\sup _{n<t \leq n+1}|R(t)-R(n)|$. Then $\left\{V_{n}\right\}$ is a sequence of independent and identically distributed random variables. We show that $E\left(V_{0}\right)<\infty$. Let $S_{j}=R\left(j 2^{-n}\right), M_{0}=0$, and $M_{k}=\max _{1 \leq j \leq k}\left|S_{j}\right|$, $k=1,2, \ldots, m=2^{n}$. For $a, b>0$, write $A_{k}=\left\{M_{k-1} \leq a+b<\left|S_{k}\right|\right\}$. Then we have $\left\{M_{m}>a+b\right\}=\cup_{k=1}^{m} A_{k}$ and $A_{j} \cap A_{k}=\emptyset \quad(j \neq k)$. So we get

$$
\begin{aligned}
P\left(\left|S_{m}\right|>a\right) & \geq \sum_{k=1}^{m} P\left(A_{k} \cap\left\{\left|S_{m}\right|>a\right\}\right) \\
& \geq \sum_{k=1}^{m} P\left(A_{k} \cap\left\{\left|S_{m}-S_{k}\right| \leq b\right\}\right) \\
& =\sum_{k=1}^{m} P\left(A_{k}\right) P\left(\left|S_{m}-S_{k}\right| \leq b\right) \\
& \geq P\left(M_{m}>a+b\right) \min _{1 \leq k \leq m} P\left(\left|S_{m}-S_{k}\right| \leq b\right) .
\end{aligned}
$$

As $P\left(M_{m} \leq b / 2\right) \leq P\left(\left|S_{m}-S_{k}\right| \leq b\right)$ is verified for $k=1, \cdots, m$, we get

$$
P\left(\left|S_{m}\right|>a\right) \geq P\left(M_{m}>a+b\right) P\left(M_{m} \leq b / 2\right) .
$$

We obtain $P(|R(1)|>a) \geq P\left(V_{0}>a+b\right) P\left(V_{0} \leq b / 2\right)$ letting $m \rightarrow \infty$. From

$$
\int_{(0, \infty)} P(|R(1)|>x) d x=\int_{(0, \infty)} x P(|R(1)| \in d x)=E(|R(1)|)
$$

and

$$
E\left(V_{0}-b: V_{0}>b\right) \leq \int_{(0, \infty)} P\left(V_{0}>x+b\right) d x \leq E(|R(1)|) / P\left(V_{0} \leq b / 2\right)
$$


(with $b$ big enough) we can conclude then $E\left(V_{0}\right)<\infty$. The strong law of large numbers implies so $\lim _{n \rightarrow \infty} \frac{V_{n}}{n}=0$. For $n \leq t<n+1$, we have

$$
\left|\frac{R(t)}{t}-E(R(1))\right| \leq\left|\frac{R(n)}{n}-E(R(1))\right|+\frac{|R(t)-R(n)|}{n}+\frac{|R(n)|}{n^{2}},
$$

and hence the conclusion.

Define $K(x)=\sum_{n=0}^{\infty} F^{n *}(x)$ for a distribution function $F$ on $(0, \infty)$. Note that for any $x>0$ there is $\ell$ such that $F^{\ell *}(x)<1$ We also have

$$
F^{n *}(x)=\int_{0}^{x} F(x-y) d F^{(n-1) *}(y) \leq F(x) F^{(n-1) *}(x) \leq F^{n}(x)
$$

for $n \geq 2$. Then, we obtain

$$
\sum_{n=0}^{\infty} F^{n *}(x) \leq \ell \sum_{k=0}^{\infty}\left(F^{\ell *}(x)\right)^{k}<\infty .
$$

Theorem 4.3 (Renewal equation). Let $F$ be a distribution function on $(0, \infty)$ and $y:[0, \infty) \rightarrow \mathbb{R}$ be a bounded Borel measurable function. Then the bounded measurable solution of the renewal equation

$$
z(x)=y(x)+\int_{0}^{x} z(x-u) F(d u)
$$

which vanishes on $x<0$ is $z(x)=\int_{0}^{x} y(x-u) K(d u)$.

Proof. Let $z_{1}, z_{2}$ be two bounded measurable solutions of (7). Then $z=z_{1}-z_{2}$ satisfies $z=z * F$. By this equality, $z=z * F^{n *}$ holds for any $n \geq 1$. Letting $n \rightarrow \infty$, we have $z(x) \leq\left(\sup _{u \geq 0} z(u)\right) F^{n *}(x) \rightarrow$ 0 . Finally, the fact that $\int_{0}^{x} y(x-u) K(d u)$ satisfies the equation (7) is straightforward.

A distribution function $F$ on $\mathbb{R}$ is called arithmetic if there is $\lambda>0$ such that the points of increase of $F$ are contained in the set $\{n \lambda: n=0, \pm 1, \pm 2, \ldots\}$. Otherwise, $F$ is non-arithmetic. 
Lemma 4.4. Let $F$ be a distribution on $(0, \infty)$ and $\Sigma$ a set formed by the points of increase of $F, F^{2 *}, F^{3 *}, \ldots$ If $F$ is non-arithmetic, then for given $\epsilon>0$ and $x$ sufficiently large the interval $(x, x+\epsilon)$ contains points of $\Sigma$.

Proof. Let $0<a<b$ be two points in the set $\Sigma$ and put $h=b-a$. Let $I_{n}=(n a, n b)=(n a, n a+n h)$. If $n>a / h,(n a,(n+1) a]$ is contained in $I_{n}$ and hence every point $x>x_{0}=a^{2} / h$ belongs to at least one among the intervals $I_{1}, I_{2}, \ldots$ The $n+1$ points $n a+k h, k=0, \ldots, n$, belong to $\Sigma$, and they partition $I_{n}$ into $n$ subintervals of length $h$. Thus every point $x>x_{0}$ is at a distance $\leq h / 2$ from a point in $\Sigma$. Suppose that there exists $\delta>0$ such that $h \geq \delta$ is satisfied for all possible choices. It follows that the points $n a+k h$ exhaust all points of $\Sigma \cap I_{n}$. Since $(n+1) a \in \Sigma$ and $(n+1) a<n b$ are satisfied, there is $1 \leq k \leq n$ for which we have $n a+a=n a+k h$ and hence $a=k h$, and thus all points of $\Sigma \cap I_{n}$ are multiples of $h$. Now let $c$ be an arbitrary point of increase of $F$. For $n$ sufficiently large the interval $I_{n}$ contains a point of the form $k h+c$, and, as this belongs to $\Sigma$, it follows that $c$ is a multiple of $h$. This shows that the distribution $F$ is arithmetic. This is a contradiction. Hence for each $\epsilon>0$ it is possible to choose $a, b$ in order to have $h<\epsilon$.

Proposition 4.5. Let $F$ be a non-arithmetic distribution function on $(0, \infty)$. If the uniformly continuous solution of

$$
z(x)=\int_{\mathbb{R}} z(x-u) F(d u)
$$

satisfies $z(x) \leq z(0)$ for every $x \in \mathbb{R}$, then $z$ is a constant.

Proof. As we have

$$
z(0)=\int_{0}^{\infty} z(-u) F^{\ell *}(d u),
$$

for $\ell=1,2, \ldots$, and $z(-u) \leq z(0)$, then $z(-y)=z(0)$ holds for $F^{\ell *}$-a.a. $y$ and, for all $\ell=1,2, \ldots$. Since $F$ is non-arithmetic, for any $\epsilon>0$ there is $x_{0}$ such that for any $x>x_{0}$ the interval $(x, x+\epsilon)$ contains a 
point of increase of $F^{\ell *}$ for some $\ell \geq 1$ (by Lemma 4.4). By the uniform continuity of $z$, we get $z(-y) \rightarrow z(0)$ as $y \rightarrow \infty$. Due to (6), for any $x \geq 0$, we have $F^{\ell *}(x) \rightarrow 0$ as $\ell \rightarrow \infty$. Hence, we get

$$
z(x)-z(0)=\int_{0}^{\infty}(z(x-u)-z(0)) F^{\ell *}(d u) \rightarrow 0
$$

as $\ell \rightarrow \infty$.

Let $f$ be a real valued function defined on $[0, \infty)$. Then $f$ is called directly integrable if the following conditions are met. If we set $m_{k}(h)=\inf _{(k-1) h \leq x \leq k h} f(x)$, plus $M_{k}(h)=\sup _{(k-1) h \leq x \leq k h} f(x)$ for $h>0$, and $s(h)=h \sum_{k} m_{k}(h), S(h)=h \sum_{k} M_{k}(h)$, then $s(h), S(h)$ are absolutely convergent and we have $\lim _{h \rightarrow 0}(S(h)-s(h))=0$.

Theorem 4.6 (Renewal theorem). Assume that $F$ is a non-arithmetic distribution function on $(0, \infty)$ and $y:[0, \infty) \rightarrow \mathbb{R}$ is directly integrable. Then for any solution $z$ of the renewal equation (7), we have that

$$
\lim _{x \rightarrow \infty} z(x)=\frac{1}{\mu} \int_{0}^{\infty} y(u) d u
$$

holds with $\mu=\int_{0}^{\infty} x F(d x)$.

Proof. The definition of $K$ gives $\int_{0}^{\infty}(K(x)-K(x-y)) F^{\ell *}(d y) \leq \ell$. For any $\alpha>0$, there is $\ell \geq 1$ such that $F^{\ell *}(\alpha)<1$. Then for $x>\alpha$ we have

$$
\ell \geq \int_{\alpha}^{\infty}\left(K(x)-K(x-y) F^{\ell *}(d y) \geq\left(1-F^{\ell *}(\alpha)\right)(K(x)-K(x-\alpha)) .\right.
$$

By this inequality, for each fixed interval $I$, we get $\sup _{t \geq 0} K(I+t)<\infty$. By Helly's selection theorem there exists a sequence $\left\{t_{k}\right\}$ and a measure $L$ on $\mathbb{R}$ such that $\lim _{k \rightarrow \infty} t_{k}=\infty$ and $K_{t_{k}}((a, b])=K\left((a, b]+t_{k}\right) \rightarrow$ $L((a, b])$ at the continuity points of $L$. Let $w$ be a continuous function which vanishes on $[0, a]^{c}$. If we set $z_{w}(x)=\int_{-\infty}^{\infty} w(x-s) K(d s)$, we get

$$
z_{w}\left(t_{k}+x\right)=\int_{-\infty}^{\infty} w(x-s) K_{t_{k}}(d s) \rightarrow \int_{-\infty}^{\infty} w(x-s) L(d s)=\zeta(x) .
$$


Note that the function $z_{w}$ satisfies the renewal equation

$$
z_{w}\left(t_{k}+x\right)=w\left(t_{k}+x\right)+\int_{0}^{\infty} z_{w}\left(t_{k}+x-s\right) F(d s) .
$$

The left hand side of the above equality tends to $\zeta(x)$ as $k \rightarrow \infty$, while the right hand side tends to $\int_{0}^{\infty} \zeta(x-s) F(d s)$ by bounded convergence. Thus $\zeta$ satisfies (8). Since $\zeta$ is bounded and uniformly continuous, by (8), we get $\zeta(x)=\zeta(0)$. So $L$ is a translation invariant measure and therefore is a multiple of Lebesgue measure. Hence there exists $\beta>0$ such that

$$
K\left(t_{k}\right)-K\left(t_{k}-h\right) \rightarrow \beta h .
$$

Further, note that

$$
1=\int_{0}^{\infty} K\left(\left(t_{k}-u, t_{k}\right]\right) F(d u) \rightarrow \beta \mu
$$

implies $\beta=\frac{1}{\mu}$. Owing to this fact, we obtain $K(t)-K(t-h) \rightarrow \frac{h}{\mu}$ as $t \rightarrow \infty$. Since $y$ is directly integrable, denoting by $M_{n}$ the supremum and by $m_{n}$ the infimum of $y(u)$ on $((n-1) h, n h]$, we have

$$
\begin{aligned}
& \sum_{n} m_{n} K\left(\left[t_{k}-n h, t_{k}-(n-1) h\right)\right) \leq z\left(t_{k}\right)=\int_{-\infty}^{0} y(-u) K_{t_{k}}(d u) \\
& \quad \leq \sum_{n} M_{n} K\left(\left[t_{k}-n h, t_{k}-(n-1) h\right)\right) .
\end{aligned}
$$

By bounded convergence, the left and right tend to $\frac{h}{\mu} \sum_{n=1}^{\infty} m_{n}$ and $\frac{h}{\mu} \sum_{n=1}^{\infty} M_{n}$, respectively, as $k \rightarrow \infty$. As $h \rightarrow 0$, each side reaches $\frac{1}{\mu} \int_{0}^{\infty} y(u) d u$.

Lemma 4.7. Let $f(x)$ be a nonnegative and non-increasing function satisfying $\int_{0}^{\infty} f(x) e^{R x} d x<\infty$ for $R>0$. Then $f(x) e^{R x}$ is directly integrable on $[0, \infty)$.

Proof. For $s(h)=h\left\{f(h)+f(2 h) e^{R h}+\cdots\right\}$ and $S(h)=h\left\{f(0) e^{R h}+\right.$ $\left.f(h) e^{2 R h}+\cdots\right\}$ we have $s(h) \leq \int_{0}^{\infty} f(x) e^{R x} d x<\infty$ and $S(h)-s(h)=$ $h f(0) e^{R h}+\left(e^{2 R h}-1\right) s(h)$, hence also $\lim _{h \rightarrow 0}(S(h)-s(h))=0$. 
Theorem 4.8. If a distribution function $F(x)$ on $(0, \infty)$ satisfies the Cramér-Lundberg condition and

$$
\mu^{*}=\frac{\lambda}{c} \int_{0}^{\infty} e^{r^{*} x} x(1-F(x)) d x<\infty
$$

holds, then

$$
\lim _{u \rightarrow \infty} e^{r^{*} u} \mathcal{E}(u)=\frac{1}{\mu^{*} r^{*}}\left(1-\frac{\lambda \mu}{c}\right) .
$$

Proof. By (4), we get

$$
\varphi(u)=\varphi(0)+\frac{\lambda}{c} \int_{0}^{u} \varphi(u-z)(1-F(z)) d z,
$$

or what is the same

$$
\begin{aligned}
1-\varphi(u)=1-\varphi(0)-\frac{\lambda}{c} \int_{0}^{u}(1-F(z)) d z \\
\quad+\frac{\lambda}{c} \int_{0}^{u}(1-\varphi(u-z))(1-F(z)) d z .
\end{aligned}
$$

From this, it follows

$$
\begin{aligned}
e^{r^{*} u} \mathcal{E}(u)=\frac{\lambda}{c} & \left(\mu-\int_{0}^{u}(1-F(z)) d z\right) e^{r^{*} u} \\
& \quad+\int_{0}^{u} \mathcal{E}(u-z) e^{r^{*}(u-z)} \frac{\lambda}{c} e^{r^{*} z}(1-F(z)) d z .
\end{aligned}
$$

Therefore the above is a renewal equation on $e^{r^{*} u} \mathcal{E}(u)$. We will now apply the renewal theorem, Theorem 4.6, to this situation. For this, note that the distribution $\frac{\lambda}{c} e^{r^{*} z}(1-F(z)) d z$ is non-arithmetic. Next, by the Cramér-Lundberg condition we get $\int_{0}^{\infty} \frac{\lambda}{c}(1-F(z)) e^{r^{*} z} d z=1$, and so we have

$$
\begin{aligned}
\frac{\lambda}{c} \int_{0}^{\infty}\left(\mu-\left(\int_{0}^{u}(1-F(z)) d z\right) e^{r^{*} u} d u\right. \\
=\frac{\lambda}{c} \int_{0}^{\infty}\left(\int_{u}^{\infty}(1-F(z)) d z\right) e^{r^{*} u} d u
\end{aligned}
$$

Pro Mathematica, 28, 55 (2014), 85-127 
Makoto Yamazato

$$
\begin{aligned}
& =\frac{\lambda}{c} \int_{0}^{\infty}(1-F(z))\left(\int_{0}^{z} e^{r^{*} u} d u\right) d z \\
& =\frac{\lambda}{c} \int_{0}^{\infty}(1-F(z)) \frac{e^{r^{*} z}-1}{r^{*}} d z \\
& =\frac{1}{r^{*}}\left(1-\frac{\lambda \mu}{c}\right) .
\end{aligned}
$$

Therefore we can apply the renewal theorem in order to get

$$
\lim _{u \rightarrow \infty} e^{r^{*} u} \mathcal{E}(u)=\frac{1}{\mu^{*} r^{*}}\left(1-\frac{\lambda \mu}{c}\right) .
$$

Example 4.9. Let $F(x)=e^{-x / \mu}$. Then the Cramér-Lundberg exponent is $r^{*}=\frac{\rho}{\mu(1+\rho)}$ and we have $\mu^{*}=\frac{c}{\lambda}$. Hence $\frac{1}{\mu^{*} r^{*}}\left(1-\frac{\lambda \mu}{c}\right)=\frac{1}{1+\rho}$. By Example 3.4, we must have $e^{r^{*} u} \mathcal{E}(x)=\frac{1}{1+\rho}$.

\subsection{Asymptotics of ruin probability (heavy tailed claims)}

In this section we discuss the case when the tail of each claim does not satisfy the Cramér-Lundberg condition. In recent years, big disasters had occurred. These phenomena imply the necessity of discussing the heavy tailed claims which cannot be treated by the Cramér-Lundberg approximation. A typical example of such heavy tailed claim is a regularly varying tail. So, at first, we explain necessary basic facts on distributions with regularly varying tails. Then we talk about subexponential distributions where the distributions with regularly varying tails are contained (see Lemma 4.25(i)). For these subexponential distributions the asymptotics of ruin probability can be easily derived. 
Non-life Insurance Mathematics

\section{Regular variation}

A measurable function $\ell:[0, \infty) \rightarrow(0, \infty)$ is called slowly varying at $\infty$ if for any $y>0$ we have

$$
\lim _{x \rightarrow \infty} \frac{\ell(x y)}{\ell(x)}=1 .
$$

We will use $h(x)=\log \ell\left(e^{x}\right)$ in what follows.

Theorem 4.10 (Uniform convergence theorem). If a function $\ell$ is slowly varying at infinity, then for any $A>0$ we have

$$
\lim _{x \rightarrow \infty} \sup _{0 \leq y \leq A}\left|\frac{\ell(x y)}{\ell(x)}-1\right|=0 .
$$

Proof. We will verify $\sup _{0 \leq u \leq A}|h(x+u)-h(x)|=0$ for a given $A>0$. Choose $\epsilon$ so that $0<\epsilon<A$ and define

$$
\begin{aligned}
I_{x} & =[x, x+2 A] \\
E_{x} & =\left\{t \in I_{x}:|h(t)-h(x)| \geq \frac{\epsilon}{2}\right\} \\
E_{x}^{*} & =\left\{t \in[0,2 A]:|h(x+t)-h(x)| \geq \frac{\epsilon}{2}\right\} .
\end{aligned}
$$

The sets $E_{x}, E_{x}^{*}$ are Borel measurable and $\left|E_{x}\right|=\left|E_{x}^{*}\right|$ (here $|\cdot|$ denotes Lebesgue measure). By the definition of slow variation, for any $y \geq 0$ we have $\lim _{x \rightarrow \infty} 1_{E_{x}^{*}}(y)=0$. Hence, the dominated convergence theorem delivers $\left|E_{x}^{*}\right| \rightarrow 0$ as $x \rightarrow \infty$. This yields the existence of $x_{0}$ for which $x \geq x_{0}$ implies $\left|E_{x}\right|<\frac{\epsilon}{2}$. For $c \in[0, A]$ we have $I_{x} \cap I_{x+c}=[x+c, x+2 A]$, while $\left|I_{x} \cap I_{x+c}\right|=2 A-c \geq A$ for $c \leq A$. In this way, for $x \geq x_{0}$, we obtain

$$
\left|E_{x} \cup E_{x+c}\right|<\left|E_{x}\right|+\left|E_{x+c}\right|=\left|E_{x}^{*}\right|+\left|E_{x+c}^{*}\right|<\epsilon<A .
$$

Hence, given $c \in[0, A]$ and $x \geq x_{0}$ we get

$$
\left|\left(I_{x} \cap I_{x+c}\right) \backslash\left(E_{x} \cup E_{x+c}\right)\right|>0 .
$$

Pro Mathematica, 28, 55 (2014), 85-127 
And we conclude $J_{x}=\left(I_{x} \cap I_{x+c}\right) \backslash\left(E_{x} \cup E_{x+c}\right) \neq \emptyset$. Since all $t \in J_{x}$ satisfies

$$
\begin{aligned}
|h(t)-h(x)| & <\epsilon / 2 \\
|h(t)-h(x+c)| & <\epsilon / 2,
\end{aligned}
$$

at the end we get $|h(x+c)-h(x)|<\epsilon$.

Lemma 4.11. If $\ell:[A, \infty) \rightarrow(0, \infty)$ satisfies

$$
\lim _{x \rightarrow \infty} \ell(x y) / \ell(x)=1
$$

for any $y>0$, then $\ell$ and $h$ are bounded in any bounded interval sufficiently far away from the origin.

Proof. By the uniform convergence theorem (Theorem 4.10) there is $x_{0}$ such that for all $x \geq x_{0}$ we have

$$
\sup _{0 \leq u \leq 1}|h(x+u)-h(x)|<1 .
$$

Hence we get

$$
\begin{aligned}
|h(x)| & \leq 1+\left|h\left(x_{0}\right)\right| \text { on }\left[x_{0}, x_{0}+1\right], \\
& \vdots \\
|h(x)| & \leq n+\left|h\left(x_{0}\right)\right| \text { on }\left[x_{0}, x_{0}+n\right] .
\end{aligned}
$$

Remark 4.12. Due to this lemma, a slowly varying function is integrable on finite intervals sufficiently far away from the origin.

Theorem 4.13 (Representation theorem). A function $\ell$ is slowly varying at infinity if and only if it can be represented as

$$
\ell(x)=c(x) \exp \left\{\int_{a}^{x} \frac{e(\log u)}{u} d u\right\} \quad(x \geq a>0),
$$

where $c$ and $e$ are measurable functions subject to $\lim _{x \rightarrow \infty} c(x)>0$ and $\lim _{x \rightarrow \infty} e(x)=0$, respectively. 
Proof. Choose $x_{0}$ large enough so that $\ell$ is integrable in any finite interval in $\left[x_{0}, \infty\right)$. For $x \geq x_{0}, h$ is written as

$$
h(x)=\int_{x}^{x+1}\{h(x)-h(t)\} d t+\int_{x_{0}}^{x}\{h(t+1)-h(t)\} d t+\int_{x_{0}}^{x_{0}+1} h(t) d t .
$$

Set $e(x)=h(x+1)-h(x)$. Then we have $\lim _{x \rightarrow \infty} e(x)=0$. From the uniform convergence theorem we get

$$
\begin{aligned}
\left|\int_{x}^{x+1}\{h(x)-h(t)\} d t\right| & =\left|\int_{0}^{1}\{h(x)-h(x+u)\} d u\right| \\
& \leq \int_{0}^{1} \sup _{0 \leq u \leq 1}|h(x)-h(x+u)| d u \rightarrow 0
\end{aligned}
$$

as $\quad(x \rightarrow \infty)$. Set $c=\int_{x_{0}}^{x_{0}+1} h(t) d t$ and $d(x)=c+\int_{x}^{x+1}\{h(x)-h(t)\} d t$. Then we have

$$
h(x)=d(x)+\int_{x_{0}}^{x} e(t) d t
$$

and so also

$$
\ell(x)=e^{h(\log x)}=e^{d(\log x)} \exp \left\{\int_{x_{0}}^{x} \frac{e(\log v)}{v} d v\right\} .
$$

Lemma 4.14 (Hamel equation). If a measurable function $f$ satisfies $f(x+y)=f(x)+f(y)$ for all $x, y \in \mathbb{R}$, then there is $c \in \mathbb{R}$ such that $f$ is represented as $f(x)=c x$.

Proof. We omit the proof. We refer the interested reader to $[3,5]$.

Theorem 4.15. Let $f:[0, \infty) \rightarrow(0, \infty)$ be measurable. Suppose that the limit

$$
g(y)=\lim _{x \rightarrow \infty} \frac{f(x y)}{f(x)}>0
$$

exists for every $y>0$. Then for some $\rho \in \mathbb{R}$ we have $g(y)=y^{\rho}$ for $y>0$. Furthermore, there exists a function $\ell$ slowly varying at infinity such that $f(x)=x^{\rho} \ell(x)$. 
Makoto Yamazato

Proof. For $y, z$ positive, we have

$$
\frac{f(x y z)}{f(x)}=\frac{f(x y z)}{f(x y)} \frac{f(x y)}{f(x)} .
$$

Therefore by taking limits we obtain $g(z y)=g(z) g(y)$. Set $h(x)=$ $\log g\left(e^{x}\right)$. Then for $x, y \in \mathbb{R}$ we get

$$
h(x+y)=\log g\left(e^{x} e^{y}\right)=\log g\left(e^{x}\right)+\log g\left(e^{y}\right)=h(x)+h(y) .
$$

By Lemma 4.14, there is $\rho \in \mathbb{R}$ such that $h(x)=\rho x$. Hence, one has $g(y)=e^{h(\log y)}=y^{\rho}$.

For the second part, it is enough to define $\ell(x)=f(x) / x^{\rho}$. In fact, as we have

$$
\begin{aligned}
\frac{\ell(x y)}{\ell(x)} & =\frac{f(x y)}{(x y)^{\rho}} \frac{x^{\rho}}{f(x)} \\
& =y^{-\rho} \frac{f(x y)}{f(x)} \rightarrow 1 \quad(x \rightarrow \infty),
\end{aligned}
$$

this function $\ell$ is slowly varying at infinity.

Remark 4.16. A positive function near infinity which satisfies the second part of the above theorem is called regularly varying at infinity with exponent $\rho$.

Lemma 4.17 (Continuity theorem). For $n \in \mathbb{N}$, let $F_{n}$ be a probability measure on $[0, \infty)$ and $\omega_{n}$ be its Laplace transform.

(1) If $\lim _{n \rightarrow \infty} \omega_{n}(s)=\omega(s)$ for $s>0$, then $\omega$ is the Laplace transform of a measure $F$ with total mass not greater than 1 and $\lim _{n \rightarrow \infty} F_{n}(x)=$ $F(x)$ for any continuity point $x$ of $F$.

(2) If $\lim _{n \rightarrow \infty} F_{n}(x)=F(x)$ holds for any continuity point $x$ of a measure $F$ with total mass not greater than 1 , then for $s>0$ we have $\lim _{n \rightarrow \infty} \omega_{n}(s)=\omega(s)$.

Proof. See [3, page 431]. 
Lemma 4.18 (Extended continuity theorem). For $n \in \mathbb{N}$, let $U_{n}$ be $a$ measure on $[0, \infty)$ and $\omega_{n}$ be its Laplace transform.

(1) If for some $a>0$ we get $\lim _{n \rightarrow \infty} \omega_{n}(s)=\omega(s)$ for any $s>a$, then $\omega$ is the Laplace transform of a measure $U$ and it satisfies $\lim _{n \rightarrow \infty} U_{n}(I)=$ $U(I)$ for any continuity interval $I$.

(2) If for any continuity interval I of $U$ conditions $\lim _{n \rightarrow \infty} U_{n}(I)=U(I)$ and $\left\{\omega_{n}(a)\right\}_{n \geq 1}$ is bounded are satisfied, then we have $\lim _{n \rightarrow \infty} \omega_{n}(s)=$ $\omega(s)$ for $s>a$.

Proof. (1) Choose $s_{0}>a$. Note that $U_{n}^{0}(d x)=\omega_{n}\left(s_{0}\right)^{-1} e^{-s_{0} x} U_{n}(d x)$ is a probability measure and its Laplace transform is $\omega_{n}\left(s+s_{0}\right) / \omega_{n}\left(s_{0}\right)$. By the continuity theorem, there exists a measure $U^{0}$ with total mass not greater than 1 such that $U_{n}^{0}$ converges to $U^{0}$. Hence $U_{n}(d x)=$ $\omega\left(s_{0}\right) e^{s_{0} x} U_{n}^{0}(d x)$ converges to $\omega\left(s_{0}\right) e^{s_{0} x} U^{0}(d x)$.

(2) Let $t>0$ be a continuity point of $U$. Then one obtains

$$
\lim _{n \rightarrow \infty} \int_{0}^{t} e^{-s x} U_{n}(d x)=\int_{0}^{t} e^{-s x} U(d x) .
$$

Furthermore, we have

$$
\int_{t}^{\infty} e^{-s x} U_{n}(d x) \leq e^{-(s-a) t} \int_{0}^{\infty} e^{-a x} U_{n}(d x)<A e^{-(s-a) t} \rightarrow 0(s>a)
$$

as $t \rightarrow \infty$, where $A=\sup _{n} \omega_{n}(a)$. For $U$, a similar property applies. Hence, one finally obtains $\lim _{n \rightarrow \infty} \omega_{n}(s)=\omega(s)$.

Theorem 4.19 (Tauberian theorem). For a non-decreasing right continuous function $U:[0, \infty) \rightarrow(0, \infty)$, the following properties

(1) $U(x) \sim c x^{\rho} \ell(x) / \Gamma(1+\rho) \quad(x \rightarrow \infty)$,

(2) $\int_{[0, \infty)} e^{-s x} d U(x) \sim c s^{-\rho} \ell(1 / s) \quad(s \downarrow 0)$,

are equivalent; here $\ell$ is slowly varying function at infinity and $c, \rho \geq 0$.

Proof. Let $\omega$ be the Laplace transform of $U$.

We first proof that (2) implies (1). By (2), one has $\frac{\omega(s t)}{\omega(s)} \rightarrow t^{-\rho}$ as $s \rightarrow 0$. Let $U_{1 / s}(x)=U(x / s)$. Then $\frac{\omega(s t)}{\omega(s)}$ and $t^{-\rho}$ are the Laplace 
transforms of the measures $\frac{U_{1 / s}(d x)}{\omega(s)}$ and $\frac{x^{\rho-1}}{\Gamma(\rho)} d x$, respectively. Applying the extended continuity theorem, one has that the measure $\frac{U_{1 / s}(d x)}{\omega(s)}$ converges to $\frac{x^{\rho}}{\Gamma(\rho)} d x$. By the assumption, we obtain $U(x) \sim c x^{\rho} \ell(x) / \Gamma(1+\rho)$.

Reciprocally, by assumption we have $\lim _{x \rightarrow \infty} \frac{U(t x)}{U(x)}=t^{\rho}$. The Laplace transform of $\frac{U(t x)}{U(x)}$ is $\frac{\omega(s / x)}{U(x)}$ and $\int_{0}^{\infty} e^{-s t} d\left(t^{\rho}\right)$ equals $s^{-\rho} \Gamma(\rho+$ 1). Dividing the interval of integration, we have $\omega(1 / x) \leq U(x)+$ $\sum_{n=1}^{\infty} e^{-2^{n-1}} U\left(2^{n} x\right)$. We can choose $x_{0}$ so that $U(2 x)<2^{\rho+1} U(x)$ for $x>x_{0}$. Thus we obtain $U\left(2^{n} x\right)<2^{n(\rho+1)} U(x)$. Using this gives

$$
\frac{\omega(1 / x)}{U(x)} \leq 1+\sum_{n=1}^{\infty} e^{-2^{n-1}} 2^{n(\rho+1)}<\infty .
$$

Hence we get $\lim _{x \rightarrow \infty} \frac{\omega(s / x)}{U(x)}=s^{-\rho} \Gamma(\rho+1)$ by the extended continuity theorem. By assumption (1), we finally have $\omega(s) \sim c s^{-\rho} \ell(1 / s)$.

\section{Subexponentiality}

Define $\bar{F}=1-F$ for a distribution function $F$ on $[0, \infty)$.

A distribution function $F$ on $[0, \infty)$ is called subexponential if it satisfies

$$
\lim _{x \rightarrow \infty} \overline{F^{2 *}}(x) / \bar{F}(x)=2 .
$$

We denote the class of subexponential distribution functions by $\mathcal{S}$.

Lemma 4.20. For $F \in \mathcal{S}$ the following two properties are satisfied.

(1) For any $A>0$, we have

$$
\lim _{x \rightarrow \infty} \sup _{0 \leq y \leq A}\left|\frac{\bar{F}(x-y)}{\bar{F}(x)}-1\right|=0,
$$

(2) For any $\epsilon>0$, we have

$$
\lim _{x \rightarrow \infty} e^{\epsilon x} \bar{F}(x)=\infty .
$$


Proof. For the first part, we fix $t \in(0, x)$ in order to have

$$
\begin{aligned}
\overline{F^{2 *}}(x) & =1-F^{2 *}(x)=1-\int_{0}^{x} F(x-y) d F(y) \\
& =1-F(x)+\int_{0}^{x} \bar{F}(x-y) d F(y) \\
& =\bar{F}(x)+\left(\int_{0}^{t}+\int_{t}^{x}\right) \bar{F}(x-y) d F(y) \\
& \geq \bar{F}(x)+\bar{F}(x) F(t)+(F(x)-F(t)) \bar{F}(x-t) .
\end{aligned}
$$

Divide both sides by $\bar{F}(x)$. Since

$$
\frac{\overline{F^{2 *}}(x)}{\bar{F}(x)} \geq 1+F(t)+\frac{(F(x)-F(t)) \bar{F}(x-t)}{\bar{F}(x)}
$$

is satisfied, we have

$$
\left(\frac{\overline{F^{2 *}}(x)}{\bar{F}(x)}-1-F(t)\right) /(F(x)-F(t)) \geq \frac{\bar{F}(x-t)}{\bar{F}(x)} .
$$

The left hand side of the above equality converges to 1 as $x \rightarrow \infty$. One has then $\limsup _{x \rightarrow \infty} \frac{\bar{F}(x-t)}{\bar{F}(x)} \leq 1$. Obviously we have $\frac{\bar{F}(x-t)}{\bar{F}(x)} \geq 1$ and therefore also

$$
\lim _{x \rightarrow \infty} \frac{\bar{F}(x-t)}{\bar{F}(x)}=1 .
$$

Since

$$
1 \leq \frac{\bar{F}(x-t)}{\bar{F}(x)} \leq \frac{\bar{F}(x-A)}{\bar{F}(x)}
$$

holds for $\leq t \leq A$, the result follows.

Now we prove the second part. By $(1)$, the distribution $\bar{F}(\log u)$ is slowly varying at infinity. By the representation theorem (Theorem 4.13), for any $\epsilon>0$ we have

$$
u^{\epsilon} \bar{F}(\log u)=e^{\int_{1}^{u} \frac{\epsilon}{v} d v} \bar{F}(\log u) \rightarrow \infty
$$

as $u \rightarrow \infty$. 
Lemma 4.21. Let $F$ be a distribution function on $[0, \infty)$. If $F(x)>0$ for any $x>0$, then we have

$$
\liminf _{x \rightarrow \infty} \frac{\overline{F^{n *}}(x)}{\bar{F}(x)} \geq n .
$$

Proof. Note that $F^{n *}(x) \leq F^{n}(x)$ holds by (6). Therefore we have

$$
\frac{\overline{F^{n *}(x)}}{\bar{F}(x)} \geq \frac{1-F^{n}(x)}{1-F(x)}=\sum_{k=0}^{n-1} F^{k}(x) \rightarrow n(x \rightarrow \infty) .
$$

Lemma 4.22. If $F \in \mathcal{S}$, then for every $n \geq 1$ we have

$$
\lim _{x \rightarrow \infty} \overline{F^{n *}}(x) / \bar{F}(x)=n .
$$

Proof. We show the result for $n=3$. First note the equalities

$$
\begin{aligned}
1-F^{3 *}(x) & =1-\int_{0}^{x} F^{2 *}(x-y) d F(y) \\
& =1-F(x)+\int_{0}^{x}\left\{1-F^{2 *}(x-y)\right\} d F(y), \\
\frac{1-F^{3 *}(x)}{1-F(x)} & =1+\int_{0}^{x} \frac{1-F^{2 *}(x-y)}{1-F(x-y)} \frac{1-F(x-y)}{1-F(x)} d F(y) .
\end{aligned}
$$

By subexponentiality of $F$ and Lemma 4.20 part (1) the integrand on the right hand side of the latter equality tends to 2 for any $y \geq 0$ as $x \rightarrow \infty$. We have for $t \in(0, x)$ then

$$
\begin{aligned}
& \left(\int_{0}^{t}+\int_{t}^{x}\right) \frac{1-F^{2 *}(x-y)}{1-F(x-y)} \frac{1-F(x-y)}{1-F(x)} d F(y) \\
\leq \sup _{x-t \leq y \leq x} & \frac{1-F^{2 *}(y)}{1-F(y)} \frac{1-F(x-t)}{1-F(x)} F(t)+K \int_{t}^{x} \frac{1-F(x-y)}{1-F(x)} d F(y),
\end{aligned}
$$

here $K=\sup _{y \geq 0} \frac{1-F^{2 *}(y)}{1-F(y)}<\infty$. Our goal is to find the limit of (9) in order to get an upper bound. Since we have $\lim _{x \rightarrow \infty} \frac{\bar{F}^{2 *}(x)}{\bar{F}(x)}=2$ and 
$\frac{\bar{F}^{2 *}(x)}{\bar{F}(x)} \leq 2$, we obtain $\sup _{x-t \leq y \leq x} \frac{1-F^{2 *}(y)}{1-F(y)} \rightarrow 2$ as $x \rightarrow \infty$. Moreover, we have

$$
\begin{aligned}
F(t) & \leq \int_{0}^{t} \frac{1-F(x-y)}{1-F(x)} d F(y) \\
& \leq \sup _{0 \leq y \leq t} \frac{1-F(x-y)}{1-F(x)} F(t) \rightarrow F(t)
\end{aligned}
$$

and

$$
\int_{0}^{x} \frac{1-F(x-y)}{1-F(x)} d F(y)=\frac{F(x)-1+1-F^{2 *}(x)}{1-F(x)} \rightarrow-1+2=1 .
$$

Hence, we get

$$
\int_{t}^{x} \frac{1-F(x-y)}{1-F(x)} d F(y) \rightarrow 1-F(t)
$$

as $x \rightarrow \infty$. By letting $x \rightarrow \infty$, the right hand side of (9) converges to $2 F(t)+K(1-F(t))$. Next, by letting $t \rightarrow \infty$, this term converges to 2 . With Lemma 4.21 at hand we conclude

$$
\frac{1-F^{3 *}(x)}{1-F(x)} \rightarrow 3 \quad \text { as } \quad x \rightarrow \infty .
$$

Lemma 4.23. If a distribution function $F$ on $[0, \infty)$ is subexponential, then for any $\epsilon>0$ there is $K>0$ such that for all $x \geq 0$ we have

$$
\overline{F^{n *}}(x) / \bar{F}(x) \leq K(1+\epsilon)^{n}, n=2,3, \ldots
$$

Proof. Define $\alpha_{n}=\sup _{x} \overline{F^{n *}}(x) / \bar{F}(x)$. Then we have

$$
\frac{\overline{F^{(n+1) *}}(x)}{\bar{F}(x)}=1+\int_{0}^{x} \frac{1-F^{n *}(x-y)}{1-F(x)} F(d y)
$$


Makoto Yamazato

$$
\begin{aligned}
\leq & 1+\sup _{x \leq M} \int_{0}^{x} \frac{1}{1-F(x)} F(d y)+ \\
& +\sup _{x \geq M} \int_{0}^{x} \frac{1-F^{n *}(x-y)}{1-F(x-y)} \frac{1-F(x-y)}{1-F(x)} F(d y) \\
\leq & 1+\frac{1}{1-F(M)}+ \\
& +\sup _{x} \frac{1-F^{n *}(x)}{1-F(x)} \times \sup _{x \geq M} \int_{0}^{x} \frac{1-F(x-y)}{1-F(x)} F(d y) \\
\leq & 1+\frac{1}{1-F(M)}+\alpha_{n}(1+\epsilon) .
\end{aligned}
$$

Where we have used that $\left|\sup _{x \geq M} \int_{0}^{x} \frac{1-F(x-y)}{1-F(x)} F(d y)-1\right|<\epsilon$ works for sufficiently big $M$. Therefore we conclude

$$
\alpha_{n+1} \leq\left(1+\frac{1}{1-F(M)}\right) \frac{1}{\epsilon}(1+\epsilon)^{n+1} .
$$

Lemma 4.24. Let $F$ be a subexponential distribution function on $[0, \infty)$. Assume that the power series $\sum_{n=0}^{\infty} a_{n} s^{n}$ is analytic at $s=1$, where $a_{n} \geq 0, \quad n=0,1,2, \ldots$ and satisfies $\sum_{n=0}^{\infty} a_{n}=1$. If we set $G(x)=$ $\sum_{n=0}^{\infty} a_{n} F^{n *}(x)$, then we get

$$
\lim _{x \rightarrow \infty} \frac{\bar{G}(x)}{\bar{F}(x)}=\sum_{n=1}^{\infty} n a_{n} .
$$

Proof. The result is obvious from Lemmas 4.22, 4.23 and the dominated convergence theorem.

Lemma 4.25. Let $F$ be a distribution function on $(0, \infty)$.

(1) If $1-F(x) \sim x^{-\rho} \ell(x)$ for a function $\ell$ slowly varying at infinity, then $F \in \mathcal{S}$.

(2) If $F$ satisfies the Cramér-Lundberg condition, then $F \notin \mathcal{S}$. 
Proof. (1) Assume that the two positive random variables $X_{1}$ and $X_{2}$ are independent with common distribution function $F$. For any $\epsilon>0$ we have

$$
\begin{aligned}
P\left(X_{1}+X_{2} \geq z\right) \leq & P\left(X_{1} \geq(1-\epsilon) z\right)+P\left(X_{2} \geq(1-\epsilon) z\right) \\
& +P\left(X_{1}>\epsilon z, X_{2}>\epsilon z\right) \\
\sim & 2(1-\epsilon)^{-\rho} z^{-\rho} \ell(z)+\left\{\epsilon^{-\rho} z^{-\rho} \ell(z)\right\}^{2} .
\end{aligned}
$$

Hence, one obtains $\limsup _{z \rightarrow \infty} \frac{P\left(X_{1}+X_{2} \geq z\right)}{P\left(X_{1} \geq z\right)} \leq 2(1-\epsilon)^{-\rho}$. Since $\epsilon$ is arbitrary, the inequality

$$
\limsup _{z \rightarrow \infty} \frac{P\left(X_{1}+X_{2} \geq z\right)}{P\left(X_{1} \geq z\right)} \leq 2
$$

follows. On the other hand, using $P\left(X_{1}+X_{2} \geq z\right) \geq P\left(X_{1} \geq z\right)+$ $P\left(X_{2} \geq z\right)-P\left(X_{1} \geq z, X_{2} \geq z\right)$ we have

$$
\liminf _{z \rightarrow \infty} \frac{P\left(X_{1}+X_{2} \geq z\right)}{P\left(X_{1} \geq z\right)} \geq 2 .
$$

(2) If $F \in \mathcal{S}$, then by Lemma 4.20, part (2), for any $\epsilon>0$ we have $e^{\epsilon x} \bar{F}(x) \rightarrow \infty$ as $x \rightarrow \infty$. So, for every $R>0$, we get $\int_{0}^{\infty} e^{R x}(1-$ $F(x)) d x=\infty$, which leads to a contradiction.

Finally we have the following characterization of the ruin probability. Recall that we have assumed $\frac{c}{\lambda \mu}>1$, i.e., $\rho>0$.

Theorem 4.26. Let $F$ be a distribution function on $(0, \infty)$ with mean $\mu<\infty$. Then $H(x)=\int_{0}^{x} \frac{1-F(y)}{\mu} d y \in \mathcal{S}$ implies

$$
\mathcal{E}(u) \sim \frac{1}{\rho} \bar{H}(u) \text { as } u \rightarrow \infty .
$$

Proof. By Theorem 3.3 we have

$$
\mathcal{E}(u)=\frac{\rho}{1+\rho} \sum_{n=0}^{\infty} \frac{\overline{H^{n *}}(u)}{(1+\rho)^{n}} .
$$


Makoto Yamazato

By Lemma 4.24 we conclude

$$
\begin{aligned}
\mathcal{E}(u) & \sim \frac{\rho}{1+\rho} \sum_{n=0}^{\infty} \frac{n}{(1+\rho)^{n}} \bar{H}(u) \\
& =\frac{1}{\rho} \bar{H}(u)
\end{aligned}
$$

as $u \rightarrow \infty$.

In contrast with the light tail case, using the previous theorem, we easily obtain the behavior as $u \rightarrow \infty$ of $\mathcal{E}(u)$ if $\bar{F}$ is regularly varying at infinity.

Acknowledgments: During the preparation of this note, Professor Kohatsu-Higa and the editor gave the author valuable advices. The author is indebted to them.

\section{References}

[1] Chistyakov, V.P.; A theorem on sums of independent positive random variables and its applications to branching processes. Theory Probability Appl. 9 (1964) 640-648.

[2] Embrechts, P., Goldie, C. M., Veraverbeke, N.; Subexponentiality and infinite divisibility, Z. Wahrscheinlichkeitstheorie verw. Gebiette 49 (1979) 335-347.

[3] Feller, W.; An Introduction to Probability Theory and its Applications, vol.2. 2nd edition., John Wiley \& Sons (1971).

[4] Kendall, D. G.; Some problems in the theory of dams, J. Roy. Statist. Soc. Ser. B. 19 (1957) 207-212.

[5] Kuczma, M.; An Introduction to the Theory of Functional Equations and Inequalities, Birkhauser, (2009), 2nd edition. 
[6] Mikosch, T.; Non-Life Insurance Mathematics, Springer (2006).

[7] Sato, K.; Lévy processes and infinitely divisible distributions, Cambridge University Press, Cambridge (1999).

\section{Resumen}

En este artículo describimos los conceptos básicos relacionados a seguros que no sean de vida y luego explicamos procesos de riesgo. En particular, tratamos al detalle el comportamiento asintótico de la probabilidad de que un producto sea declarado en ruina. Como es suponible, el comportamiento en el horizonte depende de la cola de la distribución de las primas.

Palabras clave: Procesos estocásticos, matemáticas actuariales, seguros no de vida, probabilidad de ruina, aproximación de Cramér-Lundberg.

Makoto Yamazato

Department of Mathematical Sciences,

University of the Ryukyus

Senbaru, Nishihara-cho, Okinawa 903-0213,

Japan

makotoyamazato@yahoo.co.jp 